\title{
Domain Search using Shrunken Images for Fractal Image Compression
}

\author{
Takayasu FUCHIDA, Sadayuki MURASHIMA \\ Kagoshima University \\ Hirofumi NAKAMURA \\ Miyakonojo National College of Technology
}

\begin{abstract}
In this paper, we propose a new way of limiting the number of candidates of domains by using the shrunken image for Voronoi-based fractal image compression. And we show the result of computer simulations and confirm the effects of the proposed method. The process of domain search is the most critical process of fractal image compression because it takes exorbitant time to perform it. In the process of domain search, we have to use the term of $\sum r_{i}, \sum d_{i}, \sum r_{i}^{2}, \sum d_{i}^{2}$ and $\sum r_{i} d_{i}$, where $r_{i}$ is the sum of pixels for the $i$ th range and $d_{i}$ is same one for the corresponding domain. We can calculate these terms by using cumulations for the rectangular range, but for the Voronoi range, since the shape of a range is different from each other, we can not use the cumulations for calculating these terms. Therefore, it is necessary to limit the number of candidates of domains for finding the appropriate domain in order to reduce the time of compressing image.
\end{abstract}

Key words: Fractal image compression, Voronoi diagram, Domain search, Shrunken image

\section{Introduction}

Fractal image compression is a relatively new method to compress images. The need to find solutions that can achieve optimal compression ratios with low noise at minimal computational expense attracts particular interest and attention $[1,2]$.

Fractal image compression algorithms are ones that take advantage of the self-similarity found within an image to dramatically reduce the amount of data needed to recreate the image. To find self-similarity, an image must be split up into many pieces, called the "ranges". As searching the whole image for similarity with pieces in the range is time consuming process, a smaller region is searched, known as the "domain".

The effectiveness of searching for self-similarity depends upon the ability to find an appropriate portion of the image that given a suitable affine transformation will reproduce the range image.

It is most common in research on fractal image compression that a range is simply and swiftly produced by using rectangles perhaps extended with the use of quad-tree partitioning. A range made up of rectangles has many advantageous features. It is simple to calculate the statistics of internal pixels. Position information can be expressed in a very simple form without the need for the coordinates of pixels. All of which help reducing and simplifying the computation needed in an algorithm.

Despite the advantages for using a rectangular range a nagging question remains; has the optimal range division been achieved? A rectangle might have some affinity to the physical shape of an image but no regard is given to the internal composition of an actual image. Ridged rectangles do not fit well on to the multifarious shapes found inside real images.

Irregular polygons naturally map more fittingly onto the underlying shapes observed in an image. To split an image up into irregular pentagons Voronoi division can be used. A number of points across an image are selected as kernel points. Polygons are then obtained by calculating the perpendicular bisector between all kernel points. Based on this, the principle of using Voronoi division to create a more adaptive range can be made [5]. 


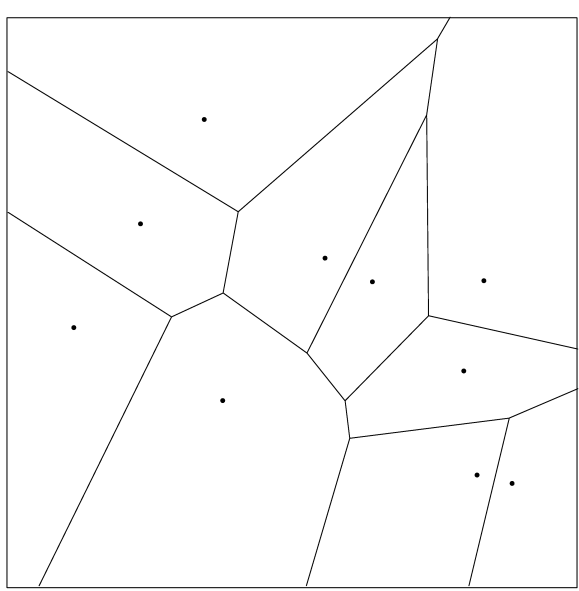

(a) continuous Voronoi diagram

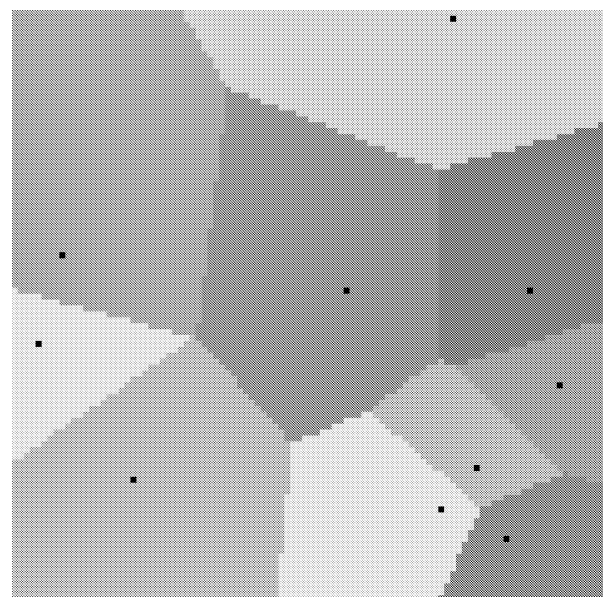

(b) discrete Voronoi diagram

Figure 1: Continuous and discrete Voronoi diagrams

A Voronoi region is more flexible but requires more calculations than a rectangular region; as the number of kernel points increases this calculation time will become excessive. But when the number of kernel points increase, in the case of using conventional discrete Voronoi division, there was a problem that this division will take a lot of time. In order to avoid this problem, we use the incremental method on a discrete space. By using this method, it became possible to perform discrete Voronoi division at so high speed, and it also became possible to use Voronoi division for fractal image compression.

In fractal image compression, the domain search is the most heavy process of all. Especially, when using Voronoi regions as ranges, since the shape of a range and a domain is complicated, a search for a suitable domain will take more time. One method of solving this problem is by restricting the number of the domains searched. In this paper, we propose a new method of using a shrunken image.

In the rest of this paper, first, the method for fractal image compression using Voronoi division is explained, and secondly, a proposal for a method to reduce the number of domains to be searched against is given. Finally the result of computer simulation are shown and the efficiency of the proposed method is confirmed.

\section{Discrete Voronoi diagram}

A Voronoi diagram is a set of irregular polygons obtained by drawing the perpendicular bisector between two kernel points. When a set of kernel points with order $k_{1}, k_{2}, \cdots, k_{N}$ is given, a Voronoi area of a kernel point $k_{i}$, which is represented by $V\left(k_{i}\right)$, is usually expressed with

$$
V\left(k_{i}\right)=\left\{p \mid d\left(p, k_{i}\right)<d\left(p, k_{j}\right) \text { for } i \neq j\right\}
$$

where $d(p, q)$ is the Euclidean distance between the points $p$ and $q$. Fig.1(a) shows an example of a continuous Voronoi diagram.

Obtaining a Voronoi diagram by calculating the perpendicular bisector between all kernel points, takes the time of $\mathrm{O}\left(N^{3}\right)$ to the number of kernel points $N$. When $N$ kernel points are given, several algorithms which construct a Voronoi diagram in the time of $\mathrm{O}(N)$ on average are commonly known.

On the other hand, some algorithms which construct a Voronoi diagram on the discrete space are also studied [4]. When an image is processed, it is more convenient to use a discrete Voronoi diagram than a continuous Voronoi diagram, because a kernel point which is contained in a region can be found by the color of the region's pixels. Fig.1(b) shows an example of a discrete Voronoi diagram.

The incremental method on the discrete space is an algorithm which constructs a discrete Voronoi diagram rapidly on a discrete space. 


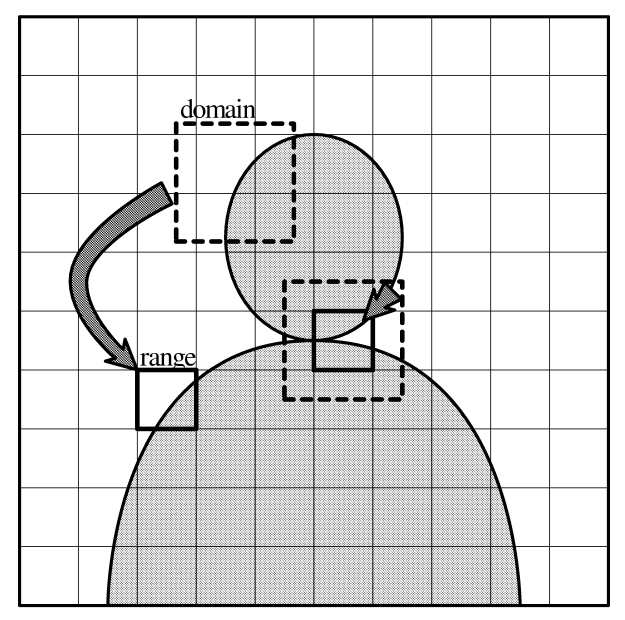

Figure 2: Affine transformation from domain to range

This algorithm efficiently constructs a new Voronoi region without disrupting surrounding areas while adding the kernel points sequentially.

In this paper, the incremental method on the discrete space is used for constructing the discrete Voronoi diagram.

\section{Fractal image compression}

The word "Fractal" was coined by the mathematician Benoit Mandelbrot [3], and the degree of irregularity and/or fragmentation of fractal shapes is identical at all scales. This feature of fractal is known as self-similarity. The fractal image compression uses this self-similarity found in a given image, and stores it into the compression file.

In this section, the processes of encoding and decoding for fractal image compression is given.

\subsection{Encoding process}

When an original image is given, it is first divided into a number of discrete areas, which do not overlap, called the ranges. For each range, a search is made for a domain whose one side is (normally) twice the length of one side of the range. A domain whose similarity to the range is the closest is searched for. The similarity is calculated using a Root Mean Square Error (RMSE) metric, as shown later. Fig.2 shows the relationship between a range and a domain. The domains may overlap with each other. Such a system like this is called partitioned iterated function system (PIFS).

The contractive map from a domain to a range can be represented by using affine transformation. General affine transformation $w_{i}$ is shown in formula (2),

$$
w_{i}\left[\begin{array}{l}
x \\
y \\
z
\end{array}\right]=\left[\begin{array}{ccc}
a_{i} & b_{i} & 0 \\
c_{i} & d_{i} & 0 \\
0 & 0 & s_{i}
\end{array}\right]\left[\begin{array}{c}
x \\
y \\
z
\end{array}\right]+\left[\begin{array}{c}
e_{i} \\
f_{i} \\
o_{i}
\end{array}\right]
$$

where $x, y$ are the coordinates of domain pixel and $z$ is the brightness of this pixel.

Parameters $a_{i}, b_{i}, c_{i}$ and $d_{i}$ determine the expansion contraction, rotation and shear, $e_{i}$ and $f_{i}$ are used for a parallel translation. Parameters $s_{i}$ and $o_{i}$ decide a brightness value.

The RMSE $E_{i}$ can be calculated as follows. Given a range containing $n$ pixels and corresponding domain containing $4 n$ pixels, and its intensities are $P_{1}^{r}, P_{2}^{r}, \cdots, P_{n}^{r}$ from range and $P_{1}^{d}, P_{2}^{d}, \cdots, P_{n}^{d}$ from domain, where $P_{i}^{r}$ is the value of the $i$ th pixel in the range $r$, and $P_{i}^{d}$ is the sum of 4 pixels of the domain $d$ corresponding to the $i$ th pixel of range $r$. The $i$ th RMSE $E_{i}$ is calculated as

$$
E_{i}=\frac{1}{n} \sum_{j=1}^{n}\left(s_{i} \cdot P_{j}^{d}+o_{i}-P_{j}^{r}\right)^{2} .
$$




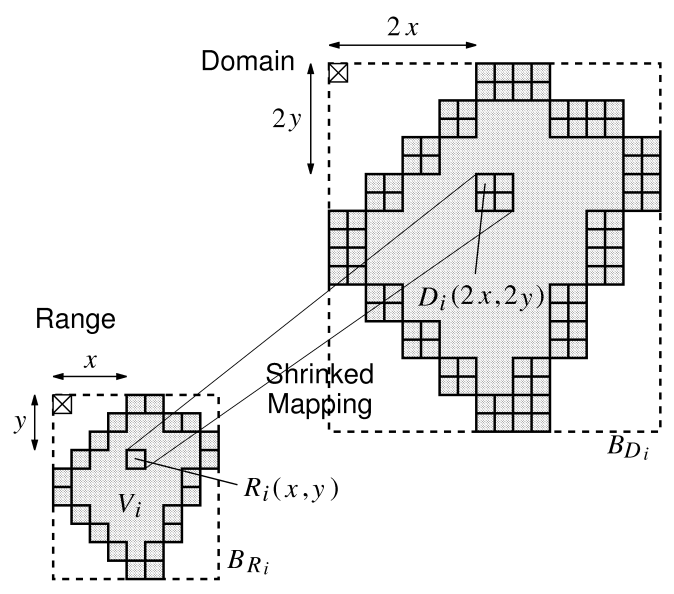

Figure 3: Shrunken mapping from domain to range

We can seek $s_{i}$ and $o_{i}$ to minimize $E_{i}$. The minimum of $E_{i}$ occurs when the partial derivatives with respect to $s_{i}$ and $o_{i}$ are zero.

$$
\frac{\partial E_{i}}{\partial s_{i}}=0, \quad \frac{\partial E_{i}}{\partial o_{i}}=0 .
$$

When the value of $E_{i}$ become settled with the smallest value, an optimum domain is found. If a domain is not found with a RMSE below the given threshold error, then the range is divided into four small rectangular parts. A new domain search will be initiated until the size of range reaches a predefined minimum value.

The ultimate step is to store in a file the chosen division of ranges, the affine parameters for each range and basic image information such as image size and pixel depth. The aforementioned is all which is needed to recover the original image because the iteration through the transformations from domains to ranges will construct the original image as explained in the next subsection.

\subsection{Decoding process}

The decoding process in fractal image compression is extremely simple compared to the preceding encoding process.

The parameters of affine transformation, division information for ranges and general image information are read first from the compressed file. Next, starting with an arbitrary initial image, the affine transformation from all domains to the corresponding ranges are repeatedly applied. At last, it converges to an image near the original image generally in 10 to 20 iterations.

The original image cannot be exactly restored because fractal image compression is essentially irreversible and lossy but a good likeness can be achieved.

\section{Fractal image compression using discrete Voronoi tessel- lation}

This section explains the use of discrete Voronoi tessellation for the fractal image compression and define some notations used, followed by a description of range division and domain searching. Finally, the encoding method for the positions of kernel points and the obtained affine transformation is given.

It is to be noted that for the affine transformation used in this research, the rate of contraction is fixed to $1 / 2$, and deformation of rotation, shear and so on, are not used.

\subsection{The definition of notations}

The pixel value at the position $(x, y)$ in the original image is expressed with $g(x, y)$, where the origin is the upper left hand corner. Width and height of the original image are expressed with $W, H$, 
respectively. The total number of ranges is $N_{R}$ and the $i$ th range is $R_{i}$ which is formed from the kernel point $k_{i}\left(k x_{i}, k y_{i}\right)$. The corresponding optimal domain for $R_{i}$ is $D_{i}$.

The minimal bounding rectangles surrounding $R_{i}$ and $D_{i}$ are $B_{R_{i}}$ and $B_{D_{i}}$ and pixel values are expressed as $R_{i}(x, y)$ and $D_{i}(x, y)$, see Fig.3.

Since the size of a domain is fixed to twice the size of a range, the pixel at $D_{i}$ corresponding to $R_{i}(x, y)$ turns into 4 pixels of $D_{i}(2 x, 2 y), D_{i}(2 x+1,2 y), D_{i}(2 x, 2 y+1)$ and $D_{i}(2 x+1,2 y+1)$. The total value of these 4 pixels is expressed with $D_{i}^{*}(x, y)$. The Voronoi region corresponding to $R_{i}$ is written $V_{i}$. Union set $V=\bigcup V_{i}$ of all $V_{i}$ expresses the whole Voronoi diagram. Moreover, $v(x, y)$ expresses which range this point belongs to. For example, if the pixel $(x, y)$ exists in the range $R_{i}$, then $v(x, y)=R_{i}$.

Linear transformation of the brightness value from $D_{i}$ to $R_{i}$ is expressed with

$$
R_{i}(x, y)=s_{i} D_{i}^{*}(x, y)+o_{i},
$$

where $s_{i}$ is called contrast and $o_{i}$ is called brightness offset.

\subsection{Range division}

Voronoi division of the range is dependent upon the position of the chosen kernel points, unlike a rectangular division of the range which is dependent upon the size of rectangle used. Choosing the location of kernel points is critical.

The best range is considered to be one whose RMSE is the smallest. Hence the following strategy is taken to decide the range division. The first division is done roughly and then iteratively, dividing those ranges which have a large RMSE.

In detail, the range division is performed according to the following steps.

1. Initially, $N_{i}$ kernel points are distributed over the whole image at random.

2. Construct Voronoi ranges and search an optimum domain for each range.

3. We rearrange the ranges sequentially in descending order of RMSE.

4. Worse $N_{w}$ ranges of RMSE are chosen and the kernel point of each range is deleted, and two new kernel points are added at random in that range.

5. Repeat step 2,3 and 4 until the number of kernel points reaches to $N_{m}$.

Fig.4 shows the process of division of an image. Fig.4(a) shows the original image "Lenna". In this example, first 500 kernel points are distributed at random over the whole image $\left(N_{i}=500\right)$ as shown in Fig.4(b). Next, the 10 ranges with the largest RMSE are improved $\left(N_{w}=10\right)$ by deleting the existing points and adding two new ones for old one. This process was repeated until the number of kernel points amounted to $2500\left(N_{m}=2500\right)$.

\subsection{Domain search}

The conversion of a pixel value from a domain to a range is calculated for all the pixels contained in $R_{i}$ using two constants $s_{i}$ and $o_{i}$ as shown in a formula (5). It is thought that this process is a linear interpolation of several points $\left(D_{i}^{*}(x, y), R_{i}(x, y)\right)$ as shown in Fig.5. Therefore, to make this error as small as possible, we have to look for a domain $D_{i}$ which makes the formula (6) minimum.

$$
E\left(s_{i}, o_{i}\right)=\sum_{x, y}\left(s_{i} D_{i}^{*}(x, y)+o_{i}-R_{i}(x, y)\right)^{2}
$$

Varying two parameters $s_{i}$ and $o_{i}$ changes the line of the interpolation. The optimum solution of a formula (6) is obtained when partial differentials about $s_{i}$ and $o_{i}$ are both set to zero as shown in formula (4), because this function is convex downward paraboloid for $s_{i}$ and $o_{i}$. This linear equation can be solved easily and the following solutions are obtained.

$$
s_{i}=\frac{n_{i} \sum R_{i} D_{i}^{*}-\sum R_{i} \sum D_{i}^{*}}{n_{i} \sum\left(D_{i}^{*}\right)^{2}-\left(\sum D_{i}^{*}\right)^{2}}
$$




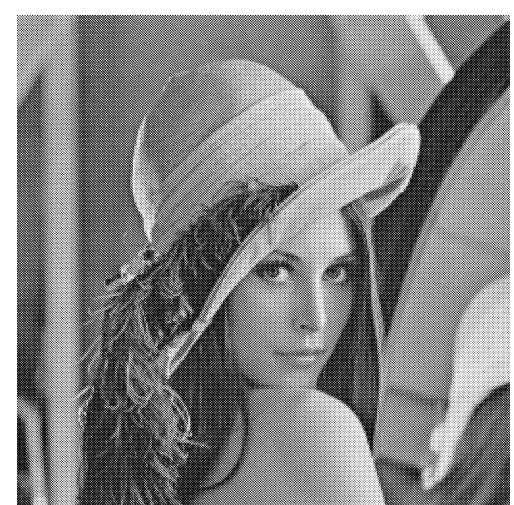

(a) Original image "Lenna"

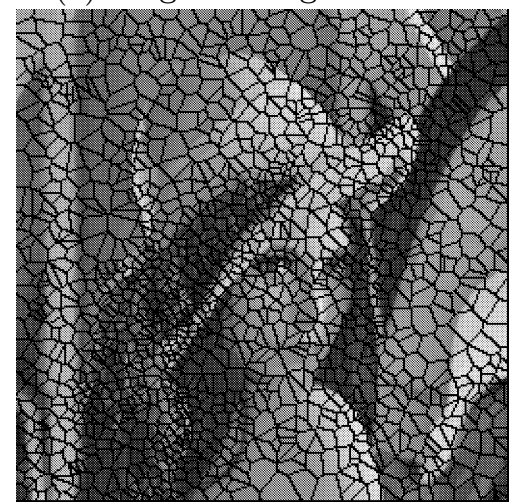

(d) 1500 kernel points

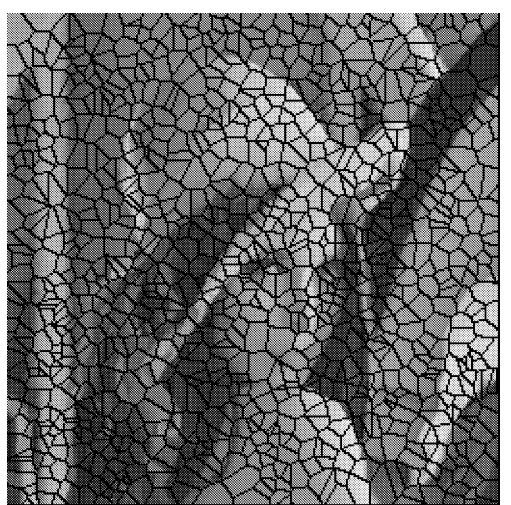

(b) 500 kernel points(initial)

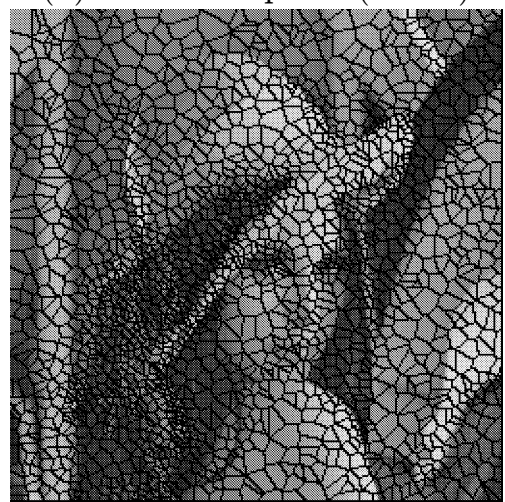

(e) 2000 kernel points

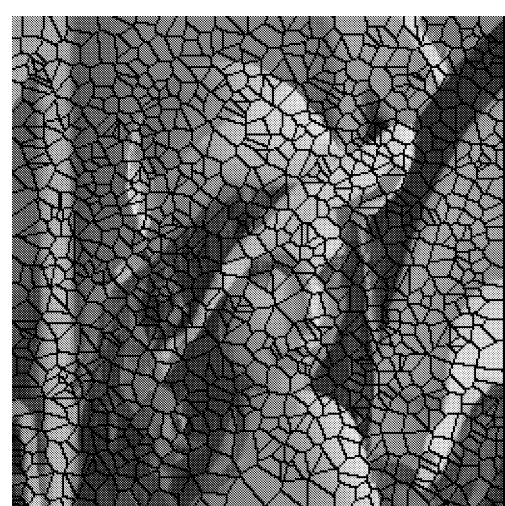

(c) 1000 kernel points

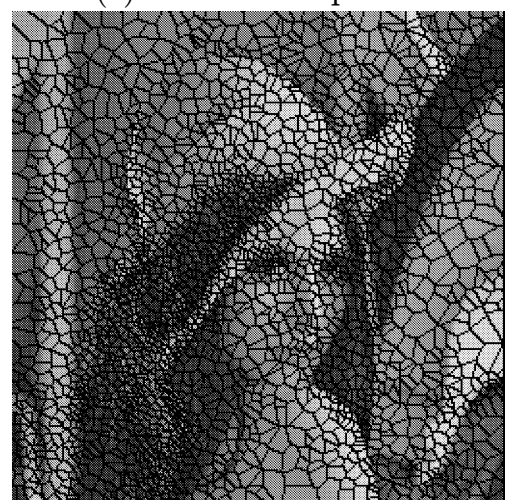

(f) 2500 kernel points

Figure 4: Process of range division

$$
o_{i}=\frac{\sum R_{i}-s_{i} \sum D_{i}^{*}}{n_{i}},
$$

where $n_{i}$ is the number of pixels contained in range $R_{i}$.

Among the parameters which appears in formulas (7) and (8), the ones only concerned with ranges such as $\sum R_{i}$ are calculable when discrete Voronoi diagram $V$ is obtained. However, since a domain is allowed to overlap, the value related to $D_{i}$ can be hardly pre-calculated.

Since the form of a Voronoi area differs for every range, each value of $\sum D_{i}^{*}, \sum\left(D_{i}^{*}\right)^{2},\left(\sum D_{i}^{*}\right)^{2}$, $\sum R_{i} D_{i}^{*}$ cannot be calculate beforehand and must be done whenever a search for a domain is made.

\subsection{Encoding of kernel point coordinates and affine transformations}

When Voronoi range division is used, the coordinates of kernel points must also be intercalated into the compressed data, as well as the domain coordinates of the affine transformations, contrasts and

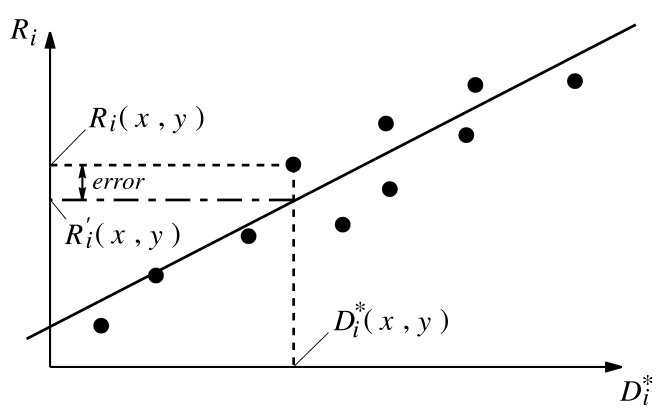

Figure 5: Linear transformation and its error 
brightness offsets.

The kernel points are distributed in a plane of the same size as the original image but the portion occupied by them is proportionally small. Hence, kernel points can be stored efficiently by using a run length code where a binary 1 is used to encode a kernel point and binary 0 for all other points. The run length code's count is stored as a Wyle's code to further save space.

The position of a domain for affine transformation is saved using the upper left coordinates of the boundary rectangle $B_{D_{i}}$ of the domain. Since the domain is twice the size of the range, if $R_{i}$ is specified, the size of $D_{i}$ corresponding to it will become settled. So it is enough to encode only its position.

The contrast and the brightness offset obtained from formulas (7), (8) are real numerical values. When storing these values to the compression file, they are quantized and stored by rounding them to integer values.

\section{Domain search using a shrunken image}

In this section, a method to reduce the computational cost of searching domains using shrunken images is proposed.

\subsection{Why does the domain search take so much time?}

The majority of time spent while processing fractal image compression is expended on the domain search. A prodigious effort is required for the following reasons; there are an exorbitant number of domains to examine, complex formula is used for calculations exasperated by the use of Voronoi range division.

The potential number of domains to be searched is immense even on a small image using rectangular range division e.g., a $256 \times 256$ image with a $16 \times 16$ domain has a potential $241 \times 241$ overlapping domains to be searched for each $8 \times 8$ range. On this case, the total number of search reaches to $241 \times 241 \times 32 \times 32$ and in approximate general terms, it is necessary to search about $N_{R} W H$ domains.

The complexity of the formula (7) and (8) needed for calculating the RMSE of a domain is especially great because the calculation of the sum of products of pixel values inside a range and a domain is included in these formulas and since it is necessary to constitute a "for" loop in the program code in order to calculate the RMSE of one domain, the search time also increases rapidly.

In addition when calculating the parameters $s_{i}$ and $o_{i}$ the rectangular frame around the Voronoi region is used. Since the Voronoi ranges are not rectangular the aforementioned "for" loop is also burdened with pixels that are in-fact out of range. (see Fig.2).

\subsection{The former method}

The easy way of reducing the number of candidates for the domain is by enlarging the unit used to incrementally shift the domain in searches (see Fig.6). The unit used during searches is given as follows, where the parameter $d s$ expresses the domain step.

$$
\text { unit }=2^{d s}
$$

For example, when $d s$ is doubled in size, the time required for domain search will decreased to $1 / 4$. If this unit is $1(d s=0)$, all domains are checked and the optimum domain will be found as a result but this has the greatest search time.

However, as the value of $d s$ becomes large, the possibility that an optimum domain will be lost also becomes large. To obtain a happy medium a method which leaves good domains and omit bad domains is required.

\subsection{Shrunken image}

The shrunken image is obtained by making the original image small by means of the Gaussian pyramid. By using the Gaussian pyramid, we can make any size of shrunken image but in this paper, we use only the quartered shrunken image which size is half in horizontally and vertically. 


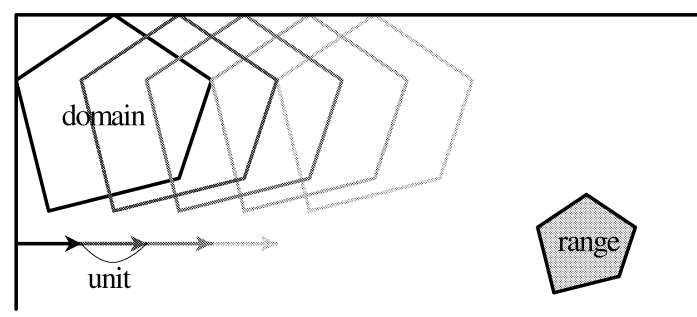

(a) the case of large $d s$.

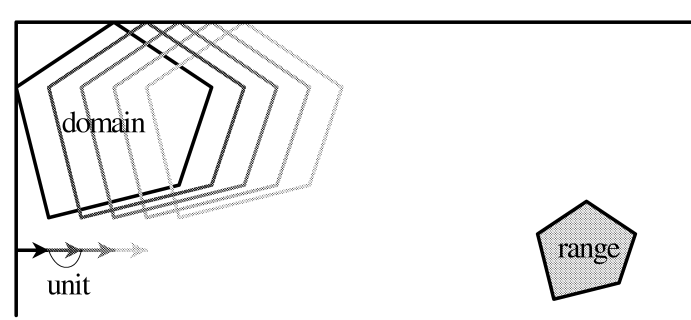

(b) the case of small $d s$.

Figure 6: Domain step of domain search

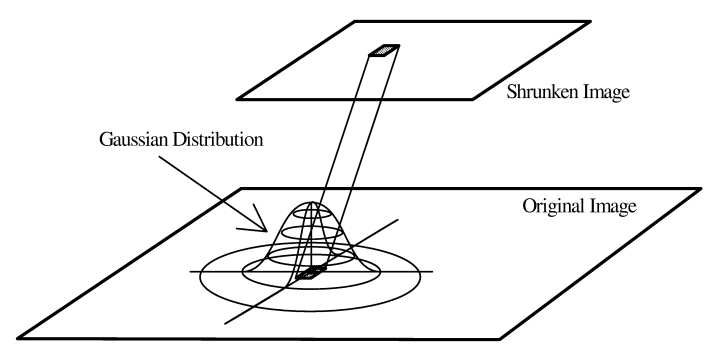

Figure 7: Shrunken image using Gaussian pyramid

An individual pixel in the shrunken image is represented as $g_{s}(x, y)$ and is calculated by following formula, the result of which is a real number.

$$
g_{s}(x, y)=\sum_{i=-2}^{2} \sum_{j=-2}^{2} w(i, j) \cdot g(2 x+i, 2 y+j)
$$

where $w(i, j)$ is the weight parameter and is given as

$$
\begin{gathered}
w(i, j)=w(i) w(j) \\
w(0)=a_{g} \\
w(1)=w(-1)=1 / 4 \\
w(2)=w(-2)=1 / 4-a_{g} / 2,
\end{gathered}
$$

where $a_{g}$ is the parameter to decide the form of Gaussian distribution. In this paper, $a_{g}$ is fixed to 0.6 .

\subsection{Domain search using shrunken image}

In order to reduce the cost of the domain search, it is important to cut off bad domains from candidates and allow the good ones to remain. A shrunken image technique is used to eliminate unlikely domains and provide a number of prospective areas worthy of detailed search.

The shrunken image, being smaller, can be searched significantly faster for candidate domains but it is possible that the position of the optimal domain in the shrunken image is incongruent with 


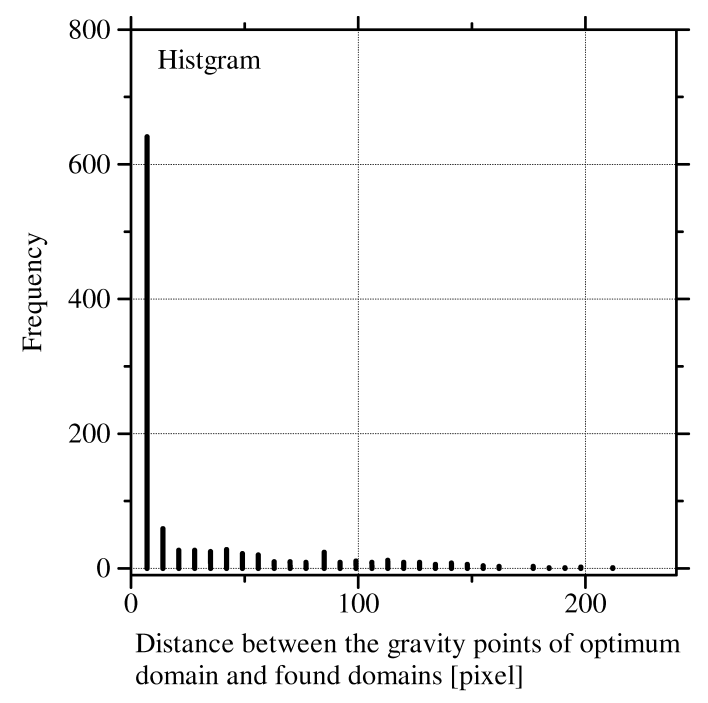

Figure 8: Histogram of distance between gravity points of optimum domain and found domains

that of the original image. Therefore several candidates of domains with the smallest RMSE are prepared.

Since the shrunken image is not the original image, any domain selected in the shrunken image is not necessarily the optimal domain. Hence, a detailed search of the surrounding domains should be made for each candidate found in the shrunken image. The detailed search is very restrictive, keeping the sum of all searches made very small, compared to a search of the whole image.

Fig. 8 shows the histogram of distance between gravity points of optimum domain and found domains in the shrunken image. This graph illustrates that most of found domains exist near by the optimum domain.

\subsection{Discrete Voronoi diagram for a shrunken image}

In order to search for a domain on the shrunken image, a shrunken Voronoi diagram has to be constructed. The shrunken Voronoi diagram must be kept discrete and similar to the original image's.

Kernel points that are adjacent to each other on the original image may well be found to be overlapping each other on the shrunken image because their coordinates are integer values. If kernel points and their Voronoi regions are lost because of overlapping then the shrunken Voronoi diagram constructed will be different for some regions.

In order to avoid losing kernel points, the shrunken discrete Voronoi diagram is made by contracting the original discrete Voronoi diagram exactly quartered. The Gaussian pyramid cannot be used to do this, as the Voronoi region must remain discrete and digital (not a real number).

Since the shrunken image size is quarter of the original image's; 4 pixels on the original discrete Voronoi diagram corresponds to 1 pixel on the shrunken Voronoi diagram (see Fig.9).

Fig.10 shows the combination of 4 pixels in the original discrete Voronoi diagram. If 3 or 4 pixels belong to the same kernel point, the shrunken pixel also belongs to that kernel point (see Fig.10(a),(b)). When the same number of pixel(s) belongs to two different kernel points, it has to be decided which kernel point the shrunken pixel is going to pertain to. In Fig.10(c), if the slashed pixels also belonged to same kernel point, this problem would occur. In this case, the shrunken pixel is forced to belong to the kernel point with smaller index. For example, if two pixels that belong to 5th kernel point and the other two belong to 10th kernel point, then the shrunken pixel belongs to 5 th kernel point.

In order to decide the kernel point for a shrunken pixel, we use the comparison values of pixels. $k_{1}, k_{2}, k_{3}$ and $k_{4}$ are the kernel points which 4 pixels of original discrete Voronoi diagram belong to (see Fig.10(d)). Then we calculate following comparison values,

$$
c_{0}=C\left(k_{1}, k_{2}\right), c_{1}=C\left(k_{1}, k_{3}\right),
$$




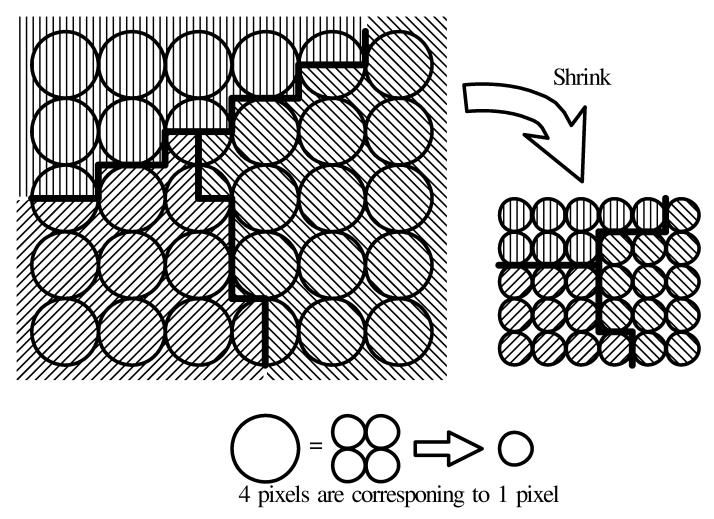

Figure 9: The way of shrinking discrete Voronoi diagram

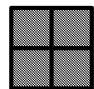

(a) all pixels belong to same kernel point.

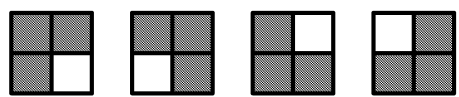

(b) only 3 pixels belong to same kernel point..

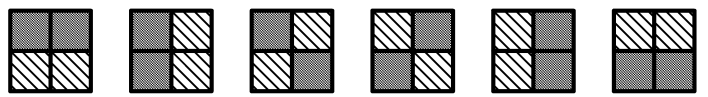

(c) 2 pixels belong to same kernel point.

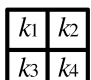

(d) no pixels belong to same kernel point.

Figure 10: Combination of the pixels of the original Voronoi diagram

$$
\begin{aligned}
& c_{2}=C\left(k_{1}, k_{4}\right), c_{3}=C\left(k_{2}, k_{3}\right), \\
& c_{4}=C\left(k_{2}, k_{4}\right), c_{5}=C\left(k_{3}, k_{4}\right),
\end{aligned}
$$

where $C\left(k_{i}, k_{j}\right)$ is the comparison function with two kernel points. if $k_{i}<k_{j}$ then $C\left(k_{i}, k_{j}\right)<0$, if $k_{i}=k_{j}$ then $C\left(k_{i}, k_{j}\right)=0$ and if $k_{i}>k_{j}$ then $C\left(k_{i}, k_{j}\right)>0$.

By using this comparison value, we can decide the kernel point of a shrunken pixel as Table 1.

\subsection{Compression algorithm}

The algorithm of domain search for range $R_{i}$ using shrunken image is as follows.

1. Read an original image.

2. Create a shrunken image using the Gaussian pyramid.

3. Distribute $N_{i}$ kernel points on the original image randomly.

4. Construct an initial discrete Voronoi diagram.

5. Build a shrunken discrete Voronoi diagram for the shrunken image using the original discrete Voronoi diagram.

6. Repeat step 7. and 8. for all range $R_{i}$.

7. Search $N_{c}$ domains which have smaller RMSE for $R_{i}$ on the shrunken image. 
Table 1: The kernel point (kp) which the shrunken pixel belongs to.

\begin{tabular}{l|l}
\hline conditions & kp \\
\hline$\left(c_{0}=c_{1}=0\right)$ or $\left(c_{1}=c_{2}=0\right)$ or $\left(c_{2}=\right.$ & $k_{1}$ \\
$\left.c_{0}=0\right)$ or $\left(c_{0}=0, c_{5}=0, c_{1}<0\right)$ or & \\
$\left(c_{0}=0, c_{5} \neq 0\right)$ or $\left(c_{1}=0, c_{4}=0, c_{0}<\right.$ & \\
$0)$ or $\left(c_{1}=0, c_{4} \neq 0\right)$ or $\left(c_{2}=0, c_{3}=\right.$ & \\
$\left.0, c_{0}<0\right)$ or $\left(c_{2}=0, c_{3} \neq 0\right)$ or $\left(c_{0}<\right.$ & \\
$\left.0, c_{1}<0, c_{2}<0\right)$ & \\
\hline$\left(c_{3}=0\right)$ or $\left(c_{4}=0\right)$ or $\left(c_{1}=0, c_{4}=\right.$ & $k_{2}$ \\
$\left.0, c_{0}>0\right)$ or $\left(c_{2}=0, c_{3}=0, c_{0}>0\right)$ or & \\
$\left(c_{3}<0, c_{4}<0\right)$ & \\
\hline$\left(c_{5}=0\right)$ or $\left(c_{0}=0, c_{5}=0, c_{1}>0\right)$ & $k_{2}$ \\
\hline others & $k_{4}$ \\
\hline
\end{tabular}

8. Search the best domain near $\pm h_{c} 2^{d s}$ area to the directions of $x$ and $y$ coordinates on the original image, where $h_{c}$ is the number of steps and $d s$ is domain step.

9. Select worse $N_{w}$ ranges from all ranges and remove kernel points of these worse ranges and add 2 kernel points randomly in every worse range.

10. Go to step 4, unless the total number of kernel points reach to $N_{m}$.

11. Save the information of kernel points, domain offsets and affine transformation parameters and image information to the compress file.

$N_{i}, N_{c}, N_{w}$ and $N_{m}$ are parameters of the number of initial ranges, the number of candidates of domains on the shrunken image, the number of worse domains and the maximum number of kernel points respectively.

\section{Computer simulations}

We performed computer simulation in order to verify the effectiveness of the proposed method.

\subsection{Simulation conditions}

The sample image is "Lenna" of a size of $512 \times 512$ pixels. The gray level at each pixel of this image is represented by 8 bits (8.0bpp image). The parameters of compression process are as follows, the domain step is $d s=2$ for normal image and $d s=1$ for shrunken image, the maximum number of range is $N_{m}=100,500,1000,2000, \cdots, 9000,10000,20000, N_{c}=3,5,10,20, h_{c}=10, N_{i}=N_{m} / 10$, $N_{w}=N_{m} / 10$.

Simulation environment: CPU was Pentium4 2.4MHz, WindowsXP Professional PC with 512MB memory. Programming language was $\mathrm{C}++$.

\subsection{Results}

Fig.11 and Fig.12 show the result of computer simulations.

The compression time is smaller than the normal method when the number of kernel points are less than 10000 and $N_{c}=3,5,10$. For $N_{c}=20$, we could not find the effectiveness but also the compression time had became larger than normal method.

Furthermore, the compression time becomes larger rapidly around the point that the number of kernel points exceeds 10,000. By the time that the number of kernel points reaches 20,000 the compression time becomes greater than using normal method for all $N_{c}$ values. 


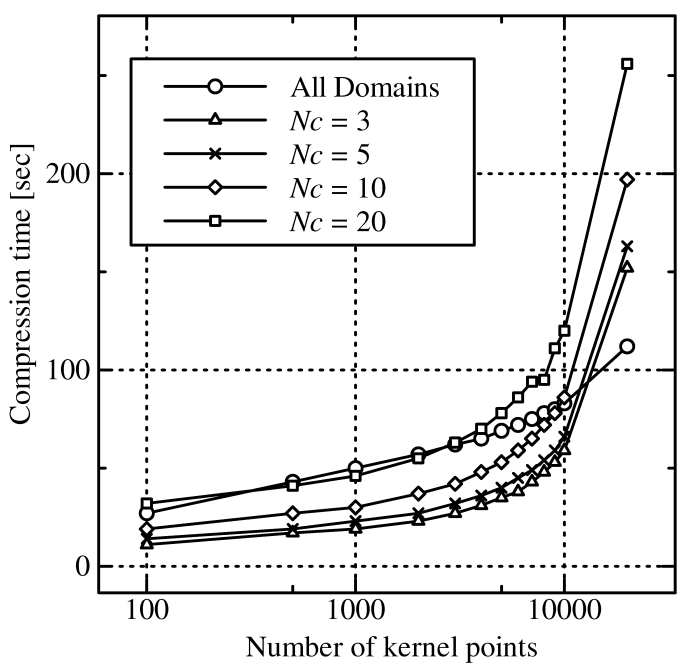

Figure 11: Compression time [sec] for various $N_{c}$

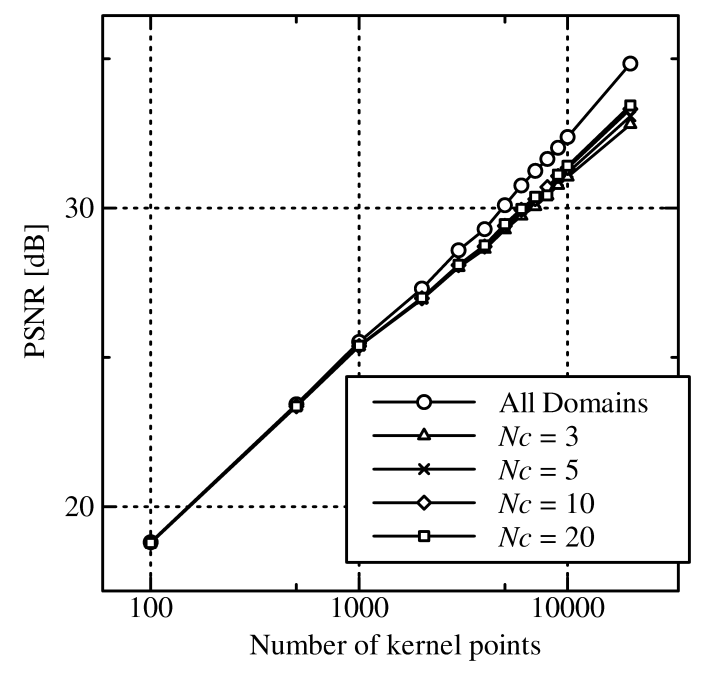

Figure 12: PSNR $[\mathrm{dB}]$ for various $N_{c}$

Quality of image is represented by Peak Signal-to-Noise Ratio (PSNR). PSNR is calculated by following equation.

$$
\begin{aligned}
\mathrm{RMSE} & =\sqrt{\frac{1}{n} \sum_{i=1}^{n}\left(g_{i}-g_{i}^{\prime}\right)^{2}} \\
\mathrm{PSNR} & =20 \log _{10}\left(\frac{255}{\mathrm{RMSE}}\right)
\end{aligned}
$$

Where $g_{i}$ is the pixel value of original image and $g_{i}^{\prime}$ is the pixel value of the restored image. $n$ is the number of pixel in the image.

The values of PSNR which indicate the quality of restored image are shown in Fig.12. From this figure, it is clear that there are few difference of PSNR values between $4 N_{c}$ s. However as the number of kernel points increase, the difference between normal method and proposal method become large and when the number of kernel points was 20,000 the difference reached is over $1.0[\mathrm{~dB}]$. This means that the optimum domain could not be found by using the proposal method. Fig.13 shows 4 of restored images of $N_{m}=500,2000,5000,10000$ for $N_{c}=3$. 


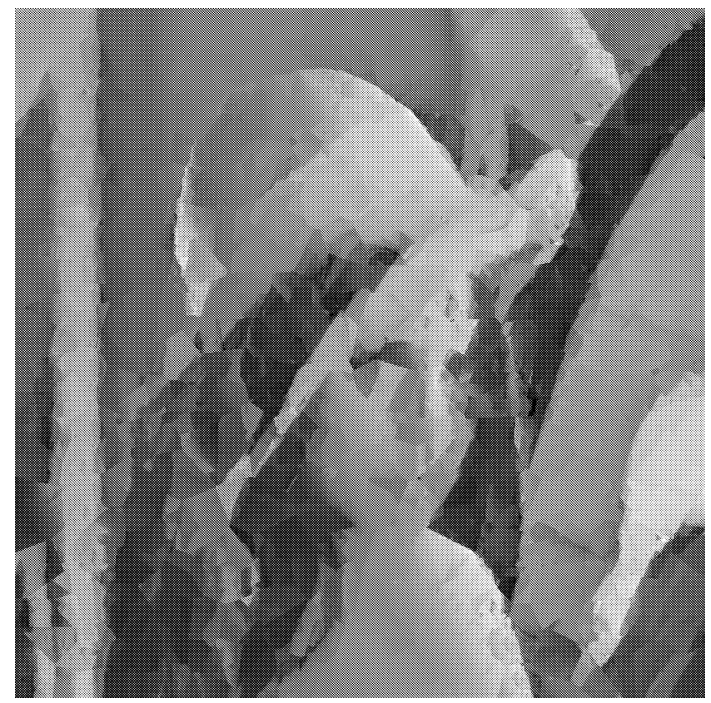

(a) $N_{m}=500(23.4 \mathrm{~dB}, 0.08 \mathrm{bpp})$

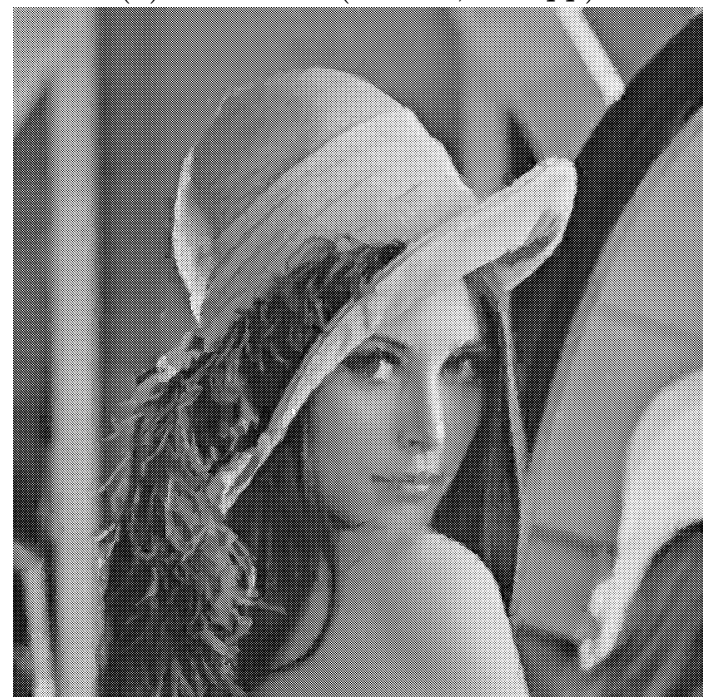

(c) $N_{m}=5000(30.1 \mathrm{~dB}, 0.66 \mathrm{bpp})$

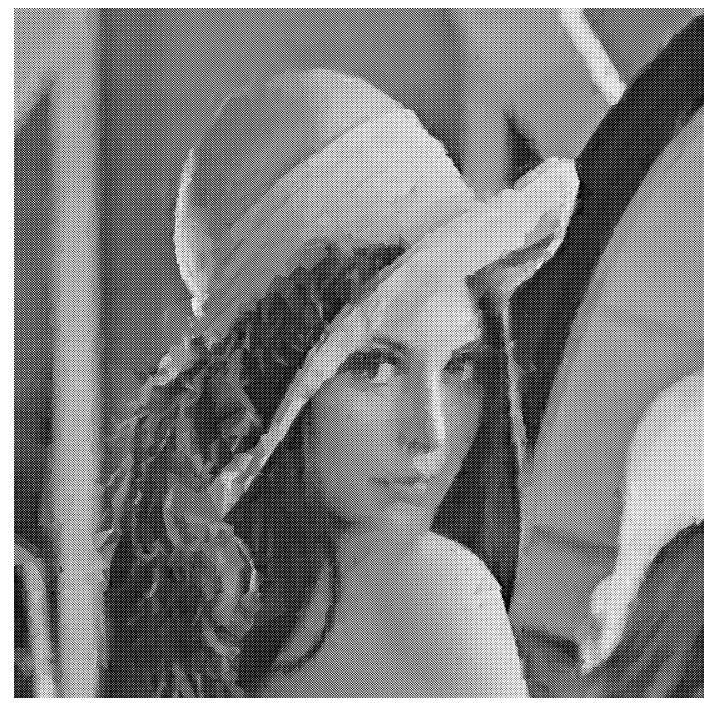

(b) $N_{m}=2000(27.3 \mathrm{~dB}, 0.29 \mathrm{bpp})$

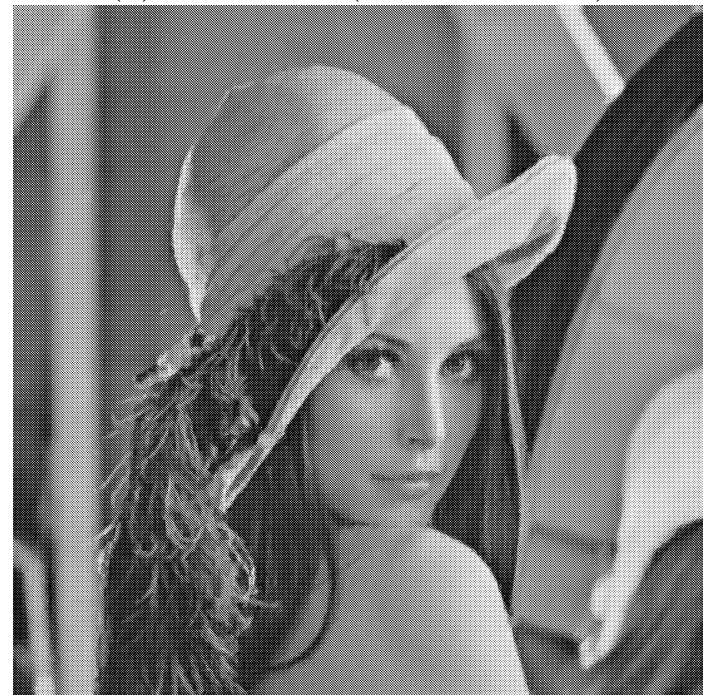

(d) $N_{m}=10000(32.4 \mathrm{~dB}, 1.24 \mathrm{bpp})$

Figure 13: Restored images for various $N_{m}$

\section{Conclusions}

This paper explains a method of fractal image compression using discrete Voronoi division, and proposed a method to improve the domain search in the compressing process.

The computer simulations show that compression time has been shortened for $N_{c}=3,5,10$ when the number of kernel points is less than 10000.

However, the compression time becomes larger rapidly when the number of kernel point exceeds 10,000. Also the quality of image is decreased by more than $1.0[\mathrm{~dB}]$.

For future work: It is intend to find out why the compression time becomes larger around the point of $N=10000$, and find a solution to decrease this time and increase the quality of the restored image.

For further thought: The splitting of Voronoi ranges, with the worse RMSE, into two has the side effect that surrounding ranges are also disrupted, even if they have good RMSE. The selection of the position of kernel points is random and takes no account of the underlying image. 


\section{Acknowledgement}

The portion of this study has been funded by the Grants-in-Aid for Scientific Research No.14780233 of the Japanese Ministry of Education, Culture, Sports, Science and Technology.

\section{References}

[1] Yuval Fisher Editor, Fractal Image Compression — Theory and Application —, Springer, 1994.

[2] M.Nelson, J.L.Gailly, The Data Compression Book Second Edition, M\&T Books, 1995.

[3] B.B. Mandelbrot, The Fractal Geometry of Nature, 2nd edition, W. H. Freeman and Co., San Francisco, California, 1982.

[4] T.Fuchida, H.Nakamura, K.Mori and S.Murashima, An Efficient Method to Construct a 2Dimensional Discrete Voronoi Diagram by Adding Kernel Points One by One, IEICE Trans A, Vol.J85-A No.5 pp.571-583, May 2002. (in Japanese)

[5] T.Fuchida, H.Nakamura, K.Mori and S.Murashima, Fractal Image Compression using Discrete Voronoi Tessellation, Proceedings of International Symposium on NOLTA 2002, Vol.2 pp.711714, Oct. 2002. 